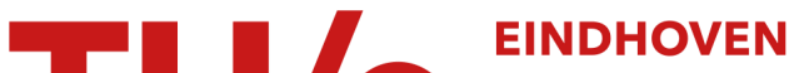 \\ UNIVERSITY OF \\ TECHNOLOGY
}

\section{Simple diffusion hopping model with convection}

\author{
Citation for published version (APA): \\ Fitzgerald, B., Padding, J. T., \& van Santen, R. A. (2017). Simple diffusion hopping model with convection. \\ Physical Review E, 95(1), [013307 ]. https://doi.org/10.1103/PhysRevE.95.013307
}

DOI:

10.1103/PhysRevE.95.013307

Document status and date:

Published: $17 / 01 / 2017$

\section{Document Version:}

Publisher's PDF, also known as Version of Record (includes final page, issue and volume numbers)

\section{Please check the document version of this publication:}

-A submitted manuscript is the version of the article upon submission and before peer-review. There can be important differences between the submitted version and the official published version of record. People interested in the research are advised to contact the author for the final version of the publication, or visit the $\mathrm{DOI}$ to the publisher's website.

- The final author version and the galley proof are versions of the publication after peer review.

- The final published version features the final layout of the paper including the volume, issue and page numbers.

Link to publication

\section{General rights}

Copyright and moral rights for the publications made accessible in the public portal are retained by the authors and/or other copyright owners and it is a condition of accessing publications that users recognise and abide by the legal requirements associated with these rights.

- Users may download and print one copy of any publication from the public portal for the purpose of private study or research.

- You may not further distribute the material or use it for any profit-making activity or commercial gain

- You may freely distribute the URL identifying the publication in the public portal.

If the publication is distributed under the terms of Article $25 \mathrm{fa}$ of the Dutch Copyright Act, indicated by the "Taverne" license above, please follow below link for the End User Agreement:

www.tue.nl/taverne

\section{Take down policy}

If you believe that this document breaches copyright please contact us at:

openaccess@tue.nl

providing details and we will investigate your claim. 


\title{
Simple diffusion hopping model with convection
}

\author{
Barry W. Fitzgerald, ${ }^{1,2, *}$ Johan T. Padding, ${ }^{2}$ and Rutger van Santen ${ }^{1}$ \\ ${ }^{1}$ Institute for Complex Molecular Systems, Eindhoven University of Technology, P.O. Box 513, 5600 MB, Eindhoven, The Netherlands \\ ${ }^{2}$ Process \& Energy Department, Delft University of Technology, Leeghwaterstraat 39, 2628 CB, Delft, The Netherlands
}

(Received 26 August 2015; revised manuscript received 8 August 2016; published 17 January 2017)

\begin{abstract}
We present results from a new variant of a diffusion hopping model, the convective diffusive lattice model, to describe the behavior of a particulate flux around bluff obstacles. Particle interactions are constrained to an underlying square lattice where particles are subject to excluded volume conditions. In an extension to previous models, we impose a real continuous velocity field upon the lattice such that particles have an associated velocity vector. We use this velocity field to mediate the position update of the particles through the use of a convective update after which particles also undergo diffusion. We demonstrate the emergence of an expected wake behind a square obstacle which increases in size with increasing object size. For larger objects we observe the presence of recirculation zones marked by the presence of symmetric vortices in qualitative agreement with experiment and previous simulations.
\end{abstract}

DOI: 10.1103/PhysRevE.95.013307

\section{INTRODUCTION}

The transportation of active and passive matter in particulate systems has been the subject of significant computational investigation in recent years. An extensively used algorithm for these studies is the particle hopping model where particles can hop between discrete cells on a lattice subject to specific movement rules or by diffusing to adjacent unoccupied cells [1]. Particle hopping models have been applied to study a variety of systems such as particle transport in disordered media [2,3], diffusion at material interfaces [4,5], the diffusion of large particles in unentangled polymer solids [6], water diffusion in cell suspension systems [7], traffic flows [8-10], ion transport through biological membranes [11,12], biological systems involving the movement of animals, micro-organisms or cells [13], and particle residency times in two-dimensional vertical strips [14].

Although hopping models allow for full control over the diffusive motion of particles, these models do not capture convective particle motion. In this paper, we introduce a variant of the lattice hopping model: the convective diffusive lattice model (CDLM). The CDLM is an extension of existing diffusion hopping models as it consists of a decoupled description of both diffusive and convective particle dynamics. Thus this model is suitable for the study of systems where diffusive behavior dominates or for processes where convective behavior is innate such as hydrodynamic flows.

In the CDLM, particle positions and dynamics are restricted to district locations on a regular underlying lattice where particles interact subject to excluded volume conditions as in previous particle hopping models $[3,4,14]$ and lattice gas methods (LGMs) which can be used to describe convective motion $[15,16]$. The main difference between previous hopping models and the CDLM is the inclusion of a continuous velocity field to capture memory bias in particle displacement, a core property of convection. In addition we describe momentum transfer with the convective dynamics.

In the CDLM, the velocity vector of the $i$ th particle $\mathbf{v}_{i}(t)$ does not necessarily represent the velocity of the particle itself, but instead represents the average, temporally and spatially

*Corresponding author: b.fitzgerald@tudelft.nl smoothed, velocity in the vicinity of particle $i$. This velocity field smoothing is similar to the implementation of kernel functions in smoothed particle hydrodynamics (SPH) [17-21] used to calculate fluid properties. The velocity field allows particles to effectively access an infinite number of particle states as dictated by the local field. This is a significant departure from traditional hopping models, where particle velocities are absent, and from the discretization of particle velocities in LGMs $[15,16]$. The underlying velocity field is evolved using an approximation of the compressible Navier-Stokes equation. Added to this, the CDLM also allows for the easy construction of complex geometries such as obstacles within flow channels. Particle interactions with any rigid boundaries or obstacles can be modeled as either no-slip or slip boundary interactions. In the case of the former, a bounce back rule is implemented preventing particles penetrating the boundaries, while for the latter, particles specularly reflect at the boundary.

This paper is arranged as follows. In Sec. II, we describe the CDLM in detail and outline the various model components such as the update sequence for the particle velocity field and positions. We also describe the implementation of boundary conditions and the parameter scalings for the model. In Sec. III A we calculate the diffusion coefficient for particles in the absence of convection. We then estimate the kinematic viscosity $v$ for a particle flow through a periodic flow channel and show the emergence of a laminar response in steady state from an initial plug velocity profile (Sec. III B). Next we compare particle transport past a square obstacle for a simulation with biased diffusion, which is the standard approach in particle hopping models, and for a system with isotropic diffusion and convective motion to demonstrate the effect of convection on particle dynamics (Sec. III C). In Sec. III D we show the emergence of recirculating vortex regions behind obstacles in a flow channel. In addition we provide evidence for the development of vortex shedding behind the obstacle at high Reynolds number. Finally we discuss our findings and suggest future explorations with the model.

\section{MODEL}

\section{A. Overview}

The CDLM is a $d$-dimensional representation of particle flow where each particle is of mass $m$ and restricted to move 
on a square lattice $\mathbf{L}$ consisting of lattice cells of side $\Delta$ and volume $\Delta^{d}$. At time $t$, particle $i$ is located at a set of discrete coordinates denoted $\mathbf{r}_{i}(t)=\left(r_{1 i}(t), \ldots, r_{d i}(t)\right)$. Particles are constrained to occupy discrete lattice volumes but move subject to a local continuous coarse-grained velocity field where the velocity for particle $i$ is $\mathbf{v}_{i}(t)=\left(v_{1 i}(t), \ldots, v_{d i}(t)\right)$. The positions and velocities of the particles are updated at every time step $\tau$. All distance measures are given in terms of $\Delta$ which is set to unity. The density of particles is defined as the lattice cell fraction $\phi_{k}$, i.e., the fraction of lattice cells occupied by particles. Further details of the scalings in the model parameters are presented in Sec. II D.

\section{B. Particle update \\ 1. Velocity update}

At each time step $\tau$, the velocity and position of each particle are updated. First, each particle is subject to a velocity update. Given that $\mathbf{v}_{i}(t)$ is associated with particle $i$, it must convectively translate with the particle. To mimic the effects that change the convective time derivative of the velocity field, we turn to the compressible Navier-Stokes equation and adapt the viscous and pressure gradient effects described in the equation. To enable spatial smoothing similar to viscous and pressure gradient effects in the Navier-Stokes expression for a particle at $\mathbf{r}_{i}(t)$, we consider the influence of all particles at lattice positions $\mathbf{r}_{i}(t)+\mathbf{e}_{\alpha}$ provided $\left|\mathbf{e}_{\alpha}\right| \leqslant R$ where the distance $R$ is large enough to sample a statistically relevant number of neighboring occupied lattice positions. Here $\mathbf{e}_{\alpha}$ is the displacement vector. The relative influence of particle located at a distance $\mathbf{r}_{i}(t)+\mathbf{e}_{\alpha}$ is controlled by a weight function $\omega_{\alpha}$. The weight function decreases monotonically with distance to reflect the declining relative influence of distant particles such that it approaches zero as $\left|\mathbf{e}_{\alpha}\right| \rightarrow R$. For the simulation results presented in this study $R=5 \Delta$. Additionally, the function should be an even function to ensure that inverse interactions are of the same strength. To meet these criteria the weight function used is given as

$$
\omega_{\alpha}\left(\mathbf{e}_{\alpha}\right)=C\left(\left|\mathbf{e}_{\alpha}\right|-R\right)^{2},
$$

where $C$ is a normalization constant such that $\int \omega_{\alpha}\left(\mathbf{e}_{\alpha}\right) d e_{\alpha}=$ 1. This integral expression is provided for convenience. More precisely, $\sum_{\alpha} \omega_{\alpha}\left(e_{\alpha}\right) \Delta^{d}$ could be set to unity when summed over all cells within $R$. However, the difference will be a factor of $O(1)$, an effect that can be encapsulated in the coefficients $A_{v}$ and $A_{c}$ associated with the velocity update. This smoothing approach is similar to smoothed particle hydrodynamics (SPH), where properties such as fluid density are calculated by smoothing over a finite volume of the fluid using kernels [17,18,20,21].

The velocity of each fluid particle is updated with the expression

$$
\begin{aligned}
\mathbf{v}_{i}(t+\tau)-\mathbf{v}_{i}(t)= & \tau A_{\nu} \sum_{\alpha} \frac{\omega_{\alpha}\left(\mathbf{e}_{\alpha}\right)}{R^{2}}\left[\mathbf{v}_{\alpha}(t)-\mathbf{v}_{i}(t)\right] \\
& -\tau A_{c} \sum_{\alpha}^{\prime} \frac{\omega_{\alpha}\left(\mathbf{e}_{\alpha}\right)}{R} \frac{\mathbf{e}_{\alpha}}{\left|\mathbf{e}_{\alpha}\right|}+\tau \mathbf{A}_{\mathbf{g}},
\end{aligned}
$$

where the prime indicates that the sum is applied only for occupied cells that are within a distance $R$ of particle $i, A_{v}$ is a term related to the kinematic viscosity of the particle flow, $A_{c}$ is related to the compressibility of the fluid, $\mathbf{A}_{\mathbf{g}}$ is related to the external fields acting on a particle, and $\mathbf{v}_{\alpha}(t)$ is the velocity of the neighboring particle at position $\mathbf{r}_{i}(t)+\mathbf{e}_{\alpha}$ at time $t$.

The first term on the right-hand side represents the viscous averaging of the velocities of the neighboring particles. The strength of this averaging effect is controlled by the viscosity term $A_{v}$. Although velocities are averaged for each particle, local momentum is conserved. The second term on the righthand side accounts for the finite compressibility of the fluid, i.e., it attenuates density fluctuations in the system. A gradient direction is approximated as the average direction to occupied neighboring lattice positions, which like the viscous term is weighted by $\omega_{\alpha}\left(\mathbf{e}_{\alpha}\right)$. Thus, occupied positions closest to $i$ will contribute to a greater extent to the local gradient. As for the first term on the right-hand side in Eq. (2), momentum is locally conserved as it is pairwise additive. The final term on the right-hand side, which includes $\mathbf{A}_{\mathbf{g}}$, accounts for any external fields, such as gravity, acting on any particle in the system. Note that we did not include a term related to thermal fluctuations. Our aim here is to model the influence of convective flows on scales much larger than the mean interparticle spacing. On such large scales thermal fluctuations are relatively unimportant. On the other hand, the effects of small-scale thermal fluctuations are explicitly modelled through diffusive position updates, as we will treat next.

\section{Position update}

The update of the particle positions consists of two distinct components: a convective update and a diffusive update. First, particles undergo convective translations based upon their respective velocity vector $\mathbf{v}_{i}$. Consider a particle at position $\mathbf{r}_{i}$ with a velocity vector $\mathbf{v}_{i}$. In the system, particles occupy rectangular cells upon a $d$-dimensional rectangular lattice $\mathbf{L}$. We propose that for a particle at $\mathbf{r}_{i}$ and with $\mathbf{v}_{i}=0$, the particle completely occupies the cell and can in fact be depicted as a "rectangular" particle. With $\left\|\mathbf{v}_{i}\right\|>0$, however, the particle will be displaced from the cell at $\mathbf{r}_{i}$ according to $\mathbf{v}_{i}$ as shown in Fig. 1 for $d=2$. Evidently, the particle no longer fully occupies the cell at $\mathbf{r}_{i}$ and for the instant shown in Fig. 1 overlaps with three neighboring cells. Given that the area of each cell is $\Delta^{d}$ and $\Delta$ is set to unity, we define $P_{i, \alpha}$, the probability of accepting a convective move to an adjacent empty cell at $\mathbf{e}_{\alpha}$, to be the area of overlap of the shifted "rectangular" particle with the neighboring cell. In addition, the degree of overlap between the displaced "rectangular" particle and the rectangular cell at $\mathbf{r}_{i}$ represents the probability of the particle remaining at $\mathbf{r}_{i}$ and is denoted as $P_{i, 0}$. Therefore, the respective probabilities satisfy the condition

$$
\sum_{\alpha=0}^{n_{\alpha}} P_{i, \alpha}=1
$$

where $n_{\alpha}$ is the number of possible destination cells including the cell $\mathbf{r}_{i}$. This approach for the implementation of convective motion does imply Galilean invariance. However, in the model presented here, the maximum permitted velocity for a particle $i$ is $\mathbf{v}_{i}^{\max }=1$. Therefore particles can move only to the nearest and next-nearest neighbors at any time step. The convective update can be adjusted to allow for $\mathbf{v}_{i}^{\max }>1$ such that a particle 


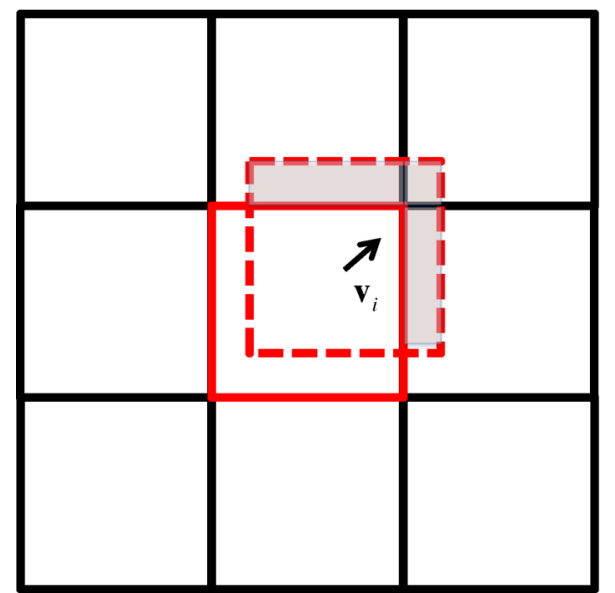

FIG. 1. Example of convective motion for a particle at cell $\mathbf{r}_{i}$ with velocity $\mathbf{v}_{i}$ on a two-dimensional grid. The dashed square represents the displaced position of the particle subject to $\mathbf{v}_{i}$. The shaded regions highlight the degree of overlap between the neighboring cells given as $\mathbf{r}_{i}+\mathbf{e}_{\alpha}$ where $\left\|\mathbf{e}_{\alpha}\right\|>0$ and the dashed square. Here $\mathbf{e}_{\alpha}$ is the displacement vector. The shaded areas are used to define displacement probabilities to the neighboring cells.

can convectively move to more cells. This adjustment may be explored with a future variant of the model.

During the convective update, it is possible that the destination cell at $\mathbf{r}_{i}+\mathbf{e}_{\alpha}$ is occupied. As a result, the particle cannot displace to the new position due to excluded volume conditions on the lattice. Rather than ignoring this convective interaction, we allow for momentum exchange between the particle moving from $\mathbf{r}_{i}$ towards the particle at $\mathbf{r}_{i}+\mathbf{e}_{\alpha}$ along the $\mathbf{e}_{\alpha}$ direction. This means that for $d=2$ for horizontal displacements, we need only exchange the velocity component along the $x$ direction, while for vertical displacements, the velocity component along the $y$ direction only needs to be adjusted. In the case of diagonal displacements, the components of the velocities along the diagonal are exchanged after which the velocity components for the particle along the $x$ direction and $y$ direction are updated. Due to the probabilistic approach used in the convective position update, the particle velocity at any position $\mathbf{r}_{i}$ must not exceed unity. Hence the parameters $A_{v}, A_{c}$, and $\mathbf{A}_{\mathbf{g}}$ in the velocity field update expression in Eq. (2) must be set accordingly.

During the diffusive update, a particle at $\mathbf{r}_{i}$ may hop to any of the four nearest neighboring cells with an equal probability $P_{i, 1}^{D}$, provided that the destination cell is empty. By considering only the four nearest neighbors, we ensure a fully isotropic diffusion tensor. For the idealised case of a single particle at $\mathbf{r}_{i}$, the diffusion coefficient $D_{0}=P_{i, 1}^{0} \Delta^{2} / \tau$.

\section{Boundary and initial conditions}

In the velocity update for a kinetic particle at $\mathbf{r}_{i}$, the influence of all other kinetic particles within a distance $R$ of $\mathbf{r}_{i}$ are considered to enable smoothing similar to viscous and pressure gradient effects. If $\mathbf{r}_{i}$ is a cell close to a boundary or obstacle, then the fraction of kinetic particles, and thus interacting particles, in its proximity will on average be less than $\phi_{k}$. For no-slip boundary conditions, particle velocity

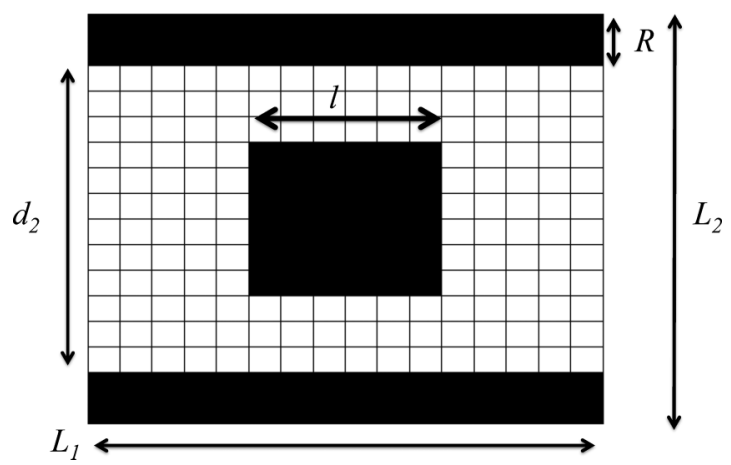

FIG. 2. Schematic of a two-dimensional system studied with the CDLM. Particle flow takes place between two no-slip rigid boundaries, each of width $R$ and length $L_{1}$. The distance between the rigid boundaries $d_{2}=L_{2}-2 R$. A bluff obstacle such as a square obstacle may be included in the flow channel. For the obstacle depicted, each side is $l$ lattice cells in length.

should approach zero close to the walls or obstacles. To include this condition, we consider an average viscous effect for boundary cells within a distance $R$ of an occupied lattice cell $\mathbf{r}_{i}$. First, we assume that all particles in the walls or obstacles are stationary. We then calculate the viscous contribution for the wall or obstacle cell at $\mathbf{r}_{i}+\mathbf{e}_{\alpha}$ using the viscous term on the right-hand side of Eq. (2). We then scale this contribution by $\phi_{k}$ thus approximating an average no-slip boundary effect at the distance $\mathbf{e}_{\alpha}$. Finally, we prohibit the penetration of particles into the walls or obstacles. When a particle attempts to move to a lattice cell that is a part of a wall or an obstacle, a bounce back rule is implemented where the velocity vector of the particle is reversed.

System boundaries and particle dynamics are confined to a rectangular two-dimensional lattice $\mathbf{L}$ where each boundary is $L_{1}$ lattice cells in length, $R$ lattice cells thick, and the distance between the boundaries is $L_{2}-2 R$ (see Fig. 2). The density of kinetic particles is set by $\phi_{k}$ with particles initially distributed randomly to empty lattice positions and the initial velocity vector at position $\mathbf{r}_{i}$ is $\mathbf{v}_{i}(t=0)=(U, 0)$. Periodic boundary conditions and an external field controlled by $\mathbf{A}_{\mathbf{g}}$ are used to sustain particle flow.

\section{System units}

In the model, the units of all measures are fixed by setting the time step $\tau$, the lattice cell size $\Delta$, and the particle mass $m$ to unity. Therefore system lengths are measured in units of $\Delta$, particle velocities are measured in units of $\Delta / \tau$, the diffusion coefficients and kinematic viscosities are measured in units of $\Delta^{2} / \tau$, system energies are measured in units of $m \Delta^{2} / \tau^{2}$, number density is measured in units of $\Delta^{-d}$, and pressure is measured in units of $m \Delta^{2-d} / \tau^{2}$.

\section{RESULTS}

\section{A. Diffusion}

For the first study with the CDLM we calculate the mean square displacement (MSD) for particles on the underlying lattice. In the absence of convection $\left(\left\|\mathbf{v}_{i}\right\|=0\right)$, the MSD in 


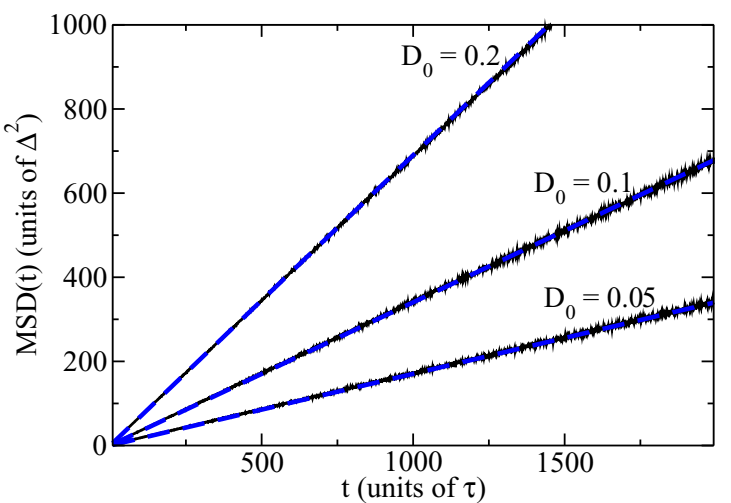

FIG. 3. Variation of the mean square displacement of particles in a fully periodic system in the absence of convective flow. Results for three different values of $D_{0}$ are shown accompanied by a straight line fit (blue dashed line) given by Eq. (4) where $D$ is a fitting parameter.

time for a particle on a $d$-dimensional lattice is given by

$$
\operatorname{MSD}(t)=\left\langle\left(\mathbf{r}_{i}(t)-\mathbf{r}_{i}(0)\right)^{2}\right\rangle=2 d D t,
$$

where $\mathbf{r}_{i}(0)$ is the initial lattice position of the particle and $\mathbf{r}_{i}(t)$ is the lattice position of the particle at time $t$. After each time step the particle may occupy any one of $n_{\alpha}$ destination cells including $\mathbf{r}_{i}$. Over a time step $\tau$, in the dilute limit, the mean square displacement for the particle at $\mathbf{r}_{i}$ to the set of $n_{\alpha}$ displacements $\mathbf{e}_{\alpha}$ is expected to be

$$
\sum_{\alpha=0}^{n_{\alpha}} P_{i, \alpha}^{D}\left|\mathbf{e}_{\alpha}\right|^{2}=2 d D_{0} \tau
$$

where $P_{i, 0}^{D}$ is the probability of the particle remaining at $\mathbf{r}_{i}$ with $\mathbf{e}_{0}=0$, and the nearest neighbors are numbered counterclockwise from 1 to $n_{\alpha}$ such that the neighbor $\alpha=1$ has the coordinates $\left(r_{1 i}+\Delta, r_{2 i}\right)$. Using Eq. (5) and given that for $\alpha>0\left|\mathbf{e}_{\alpha}\right|=1$, and all $P_{i, \alpha}^{D}$ are equal for $1 \leqslant \alpha \leqslant 4$, we can write

$$
4 P_{i, 1}^{D}=4 D_{0} \tau
$$

Therefore, in the dilute limit, the diffusion coefficient $D_{0}=$ $P_{i, 1}^{D} / \tau$. Figure 3 shows the variation of $\operatorname{MSD}(t)$ for three different values of $D_{0}$ in a fully periodic system of side $L_{1}=L_{2}=1000 \Delta$ where the density of kinetic particles $\phi_{k}=0.1$. Each curve is fitted with a straight line given by Eq. (4). In the case where the input $D_{0}=0.1 \Delta^{2} / \tau$, the simulations yield a finite concentration diffusion coefficient $D=0.086 \Delta^{2} / \tau$, a value that is in agreement with the nonzero density of kinetic particles in the system. Given a particle at the origin, the likelihood of jumping to a neighbor is $4 P_{i}^{D}\left(1-\phi_{k}\right)$. Therefore the effective finite concentration diffusion coefficient $D=D_{0}\left(1-\phi_{k}\right)=0.09 \Delta^{2} / \tau$. A smaller effective diffusion coefficient has also been measured with lattice gas models [22,23].

\section{B. Convection}

In the CDLM the parameter $A_{v}$ is related to the kinematic viscosity $v$ of the fluid. In this section we estimate the value of $v$ within the bulk of the particle field. For each configuration the lattice cells between the rigid boundaries are

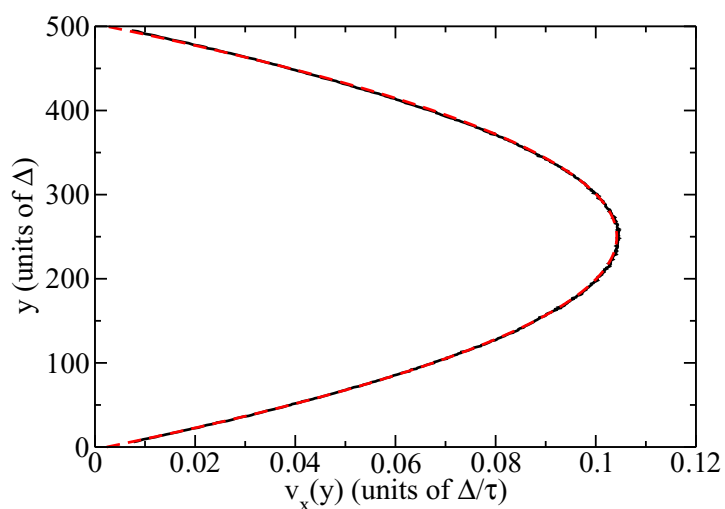

FIG. 4. Spatiotemporally averaged velocity profile (black) calculated in steady state for a system with $L_{1}=500 \Delta$ and $L_{2}=500 \Delta$. The dashed line (red) is a fit given by Eq. (7) to the simulation data.

randomly occupied with kinetic particles such that $\phi_{k}=0.1$. The initial velocity of each particle is $\mathbf{v}_{i}(t=0)=(U, 0)$ where $U=0.1 \Delta / \tau$, thus representing a uniform plug flow over the width of the flow channel. Upon initiation of the flow the no-slip boundary conditions result in the dissipation of particle energy due to the friction experienced by particles adjacent to the boundary. This in turn leads to further dissipation for particles in the adjacent layers and thus produces two boundary layers associated with each of the rigid boundaries. By means of an external field the particles are kept in motion. As a result a maximum in the velocity flow field is expected in the center of the flow channel such that a parabolic velocity profile typifying a Hagen-Poiseuille or laminar flow emerges.

Figure 4 shows a spatiotemporally averaged velocity profile for a square flow channel with $L_{1}=L_{2}=500 \Delta$. For this simulation $A_{v}=1 \Delta^{2} / \tau, A_{c}=0.01 \Delta^{2} / \tau^{2}, A_{g, x}=$ $1.54 \times 10^{-7} \Delta / \tau^{2}$, and $D=0.05 \Delta^{2} / \tau$. The velocity profile is spatially averaged along the length of the flow channel once a steady-state flow has developed and then temporally averaged over a time of $5 \times 10^{4} \tau$ in steady state. The velocity profile has been fitted with an equation of the form

$$
v_{x}(y)=\frac{d_{2}^{2} A_{g, x}}{8 v}\left[1-4\left(\frac{y}{d_{2}}\right)^{2}\right] .
$$

From the fit to the velocity profile in Fig. 4 we find $v=$ $0.0462 \Delta^{2} / \tau$.

We have also explored the effect of system size on $v$. The main figure of Fig. 5 shows the variation of $v$ with $L$ where $L=L_{1}=L_{2}$. With increasing $L$ we observe that $v$ quickly converges towards a value that is representative of an infinitely large flow channel. The simulation data is accompanied by a fit of the form $v(L)=v_{\infty}[1-\exp (-\alpha L)]$ where $v_{\infty}=0.0462 \pm$ 0.0004 , the kinematic viscosity expected for a flow channel of infinite extent. We also plot the variation of $A_{g, x}$, the external field needed to achieve $U_{\max }=0.1 \Delta / \tau$ with $L$ as shown in the inset figure of Fig. 5. The simulation data can be fitted with an expression of the form $A_{g, x}(L)=C_{A g} L^{\beta}$ where $\beta=$ $-1.98 \pm 0.01$, which is expected given the form of Eq. (7). Similar trends can be calculated with different values of $U, D$, and $A_{c}$. 


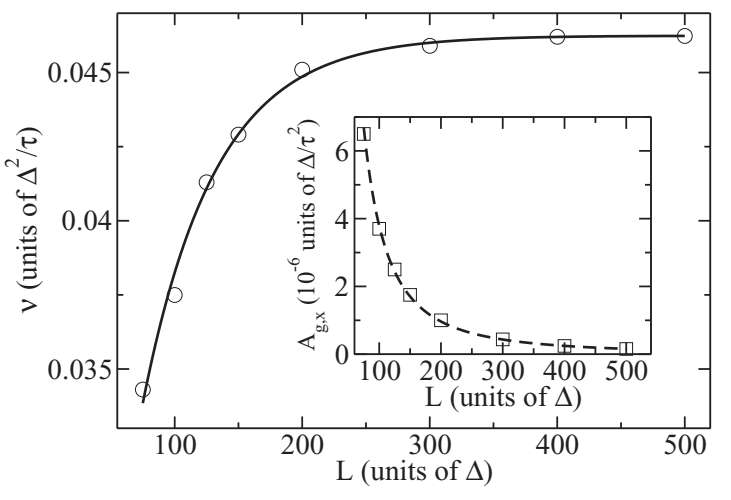

FIG. 5. Variation of $v$ with $L$. The open circles are the simulation data, while the solid black line is a fit given by $v(L)=v_{\infty}[1-$ $\exp (-\alpha L)]$ where $v_{\infty}=0.0462 \pm 0.0004$ and $\alpha=0.0176 \pm 0.0001$. (Inset) Variation of $A_{g, x}$ with $L$, the external field required to achieve a constant flow velocity $U_{\max }$. The open squares are the simulation data, while the dashed black line is a fit given by $A_{g, x}(L)=C_{A g} L^{\beta}$ where $C_{A g}=0.034 \pm 0.001$ and $\beta=-1.98 \pm 0.01$. For both figures $L=L_{1}=L_{2}$.

In addition we have varied the diffusion coefficient to ascertain its affect on $v$. Figure 6 shows the variation of $v$ with $D$ for a system with $L_{1}=L_{2}=300 \Delta$. We note that $v$ increases with increasing $D$ due to the creation of additional momentum transfer in the fluid by random displacements of the particles. The increase in $v$ with $D$ is best described by a fit given by $\nu(D)=E_{v} D^{\epsilon}$ where $\epsilon=0.582 \pm 0.002$. In addition Fig. 6 indicates that the Schmidt number $\mathrm{Sc}=v / D \approx 1$, for all values of $D$. The relationship between $v$ and $D$ is very much dependent on the choice of the weight function or smoothing kernel and the nature of the diffusive update. An alternative form of the weight function [Eq. (1)], variation of $R$ or adjusting the the number of possible destination sites during the diffusive update can all be used to adjust the resultant kinematic viscosity and thus lead to $\mathrm{Sc}>1$.

\section{Biased diffusion versus convective dynamics}

To demonstrate the qualitative differences due to convective dynamics on particle flow, we compare CDLM simulations for cases with and without convective dynamics. While CDLM

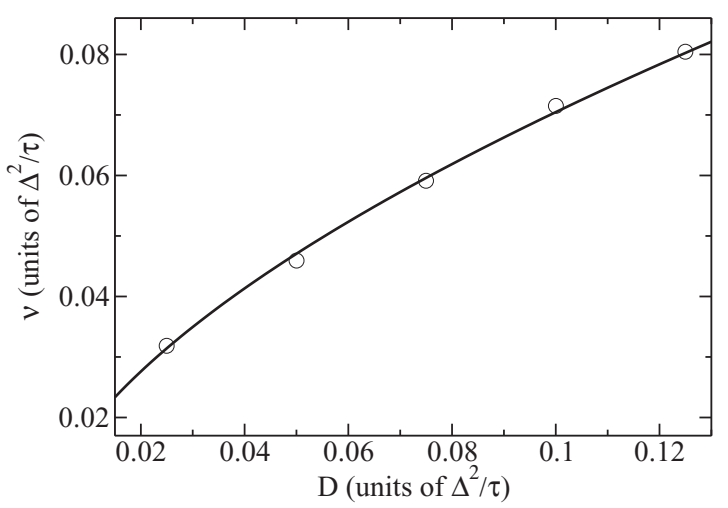

FIG. 6. Variation of $v$ with $D$ for a flow channel with $L_{1}=L_{2}=$ $300 \Delta$. The solid black line is a power law fit to the data such that $v(D)=E_{v} D^{\epsilon}$. Here $E_{v}=0.269 \pm 0.001$ and $\epsilon=0.582 \pm 0.002$. convective dynamics ensures particle motion using an external body force, the net transport of particles from the inlet to outlet region in traditional diffusion hopping systems is sustained by implementing a biased diffusion where the probability for a particle to diffuse to the right $P_{i, 1}^{D}=D_{1}$ and the probability for all other destination cells is $D_{0}$. This approach is similar to that used in studies of residency time on vertical strips [14] and in driven diffusive systems [24] where particles can be argued to move from an inlet region to an outlet region subject to an external potential field.

For the simulations with convective dynamics, we set $U=0.1 \Delta / \tau, A_{v}=1 \Delta^{2} / \tau, A_{c}=0.01 \Delta^{2} / \tau^{2}$, and $A_{g, x}=$ $8 \nu U / d_{2}^{2} \Delta / \tau^{2}$. In the previous section we calculated $v=$ $0.0462 \Delta^{2} / \tau$ for these parameters. In addition we restore isotropic diffusion such that $D_{0}=0.05 \Delta^{2} / \tau$. For both model cases, $\phi_{k}=0.1$.

To allow for the direct quantitative comparison of simulations with and without convective dynamics, we study particle transport for simulations at the same Peclet number (Pe) as calculated in terms of the obstacle size $l$ with

$$
\mathrm{Pe}=\frac{\bar{v} l}{D_{0}},
$$

where $\bar{v}$ is the average velocity at the center of the flow channel and $D_{0}$ is the diffusion coefficient. For the simulations including convective motion, we denote the Peclet number as $\mathrm{Pe}_{c}$ and set $\bar{v}=U$, the average particle velocity at the center of an empty flow channel. The dimensions of the flow channel are $L_{1}=1000 \Delta$ and $L_{2}=500 \Delta$. Hence for a system containing a square obstacle of side $l=200 \Delta$, from Eq. (8), $\mathrm{Pe}_{c}=320$. To ensure that the Peclet number for the biased diffusion simulations $\mathrm{Pe}_{b}$ is equal to the Peclet number for simulations including convective motion, we calculated $\bar{v}_{b}$, the average diffusive particle velocity at the center of an empty flow channel for varying values of $D_{1}$ such that $D_{1}>D_{0}$. Best correspondence was found for $D_{1}=0.11 \Delta^{2} / \tau$ such that $\bar{v}_{b}=0.1 \Delta^{2} / \tau$ and, as a result $\mathrm{Pe}_{b}=\mathrm{Pe}_{c}$.

Figure 7 shows color maps for the particle occupancy in steady state for a system with biased diffusion and a system with convective dynamics in combination with isotropic diffusion. For a lattice cell with coordinate center $\mathbf{r}_{i}(t)$, the average particle occupancy $\psi_{\mathbf{r}_{i}}$ is the fraction of times that a particle occupied the cell over a time window $\tau_{w}$ such that

$$
\psi_{\mathbf{r}_{i}}=\frac{1}{\tau_{w}} \sum_{t=1}^{\tau_{w}} \Psi(t)_{\mathbf{r}_{i}},
$$

where $\Psi(t)_{\mathbf{r}_{i}}=1$ if a cell is occupied and $\Psi(t)_{\mathbf{r}_{i}}=0$ if a cell is unoccupied at time $t$.

In Figs. 7(a) and 7(b) the average particle occupancy at each cell is normalized by the maximum particle occupancy $\psi_{\max }$ over a time window $\tau_{w}=5 \times 10^{4} \tau$ such that $\psi_{\mathbf{r}_{i}}^{n}=\psi_{\mathbf{r}_{i}} / \psi_{\max }$ where $\psi_{\max }=\tau_{w}$. The limits for the gradient scale for the map are such that a cell with $\psi_{\mathbf{r}_{i}}^{n}=0$ is black and a cell with $\psi_{\mathbf{r}_{i}}^{n}=1$ is white. For the system with biased diffusion the color map shows particle build up on the left side of the obstacle. Although particles can diffuse around the object, the color map reveals that particles rarely enter, if at all, the region directly behind the obstacle. In comparison, the simulation with convective dynamics [Fig. 7(b)] shows a moderate buildup of particles on the left side of the obstacle where 


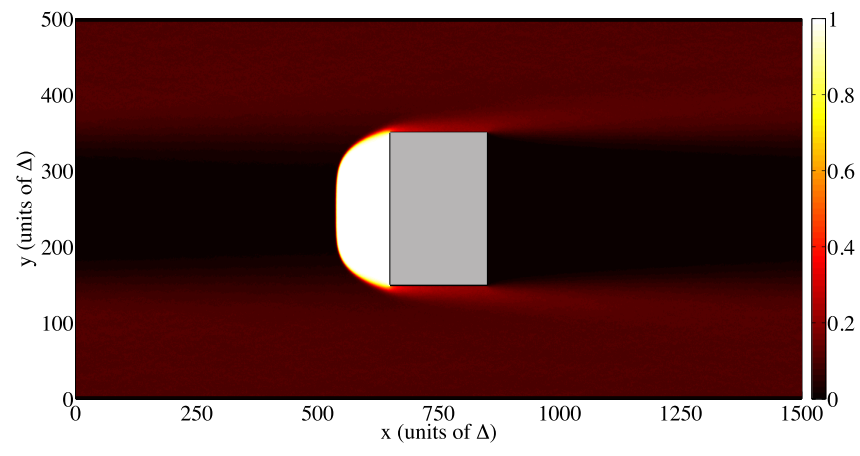

(a)

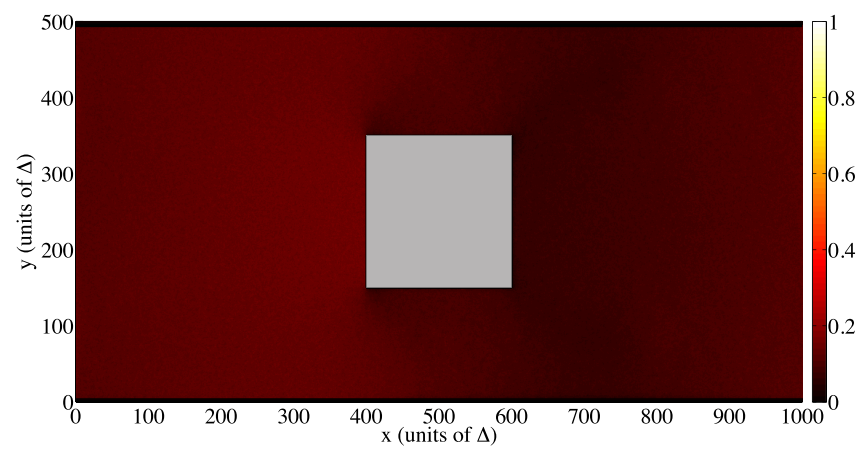

(b)

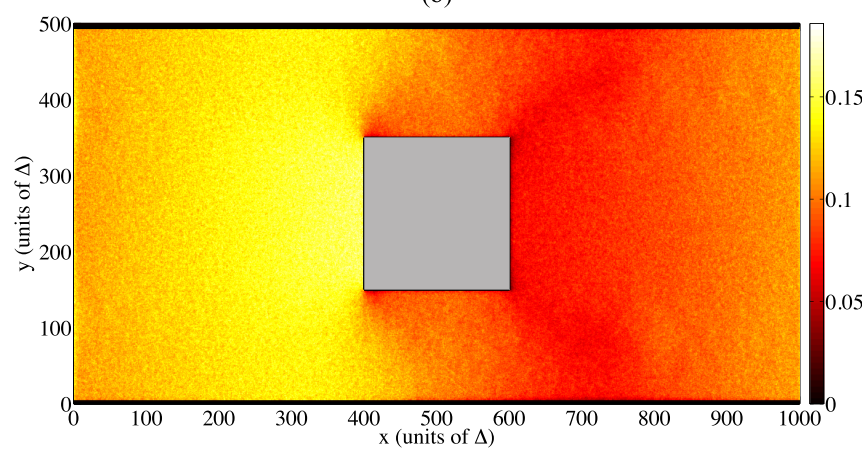

(c)

FIG. 7. Color maps for average particle occupancy during steadystate flow in a flow channel containing a square obstacle of side $l=200 \Delta$ with $\tau_{w}=5 \times 10^{4} \tau$. (a) Biased diffusion where $L_{1}=$ $1500 \Delta$ and $L_{2}=500 \Delta$. (b) Convective motion where $L_{1}=1000 \Delta$ and $L_{2}=500 \Delta$. (c) Convective motion where $L_{1}=1000 \Delta$ and $L_{2}=500 \Delta$ (alternative scaling for color map). For this particle flow the Reynolds number $\mathrm{Re} \approx 650$ as calculated using Eq. (10).

$\psi_{\mathbf{r}_{i}}^{n} \approx 0.2$. Beyond the obstacle we note a nonzero particle occupancy such that $\psi_{\mathbf{r}_{i}}^{n}<0.2$. To better resolve the color map for the simulation with convective dynamics, we have replotted the color map from Fig. 7(b) without normalization of the particle occupancy with $\psi_{\max }$. The revised color map is shown in Fig. 7(c). This figure clearly demonstrates a build up of particles on the left side of the obstacle where $\psi_{\mathbf{r}_{i}}^{n}>\phi_{k}$ and a wake region to the right of the obstacle where $\psi_{\mathbf{r}_{i}}^{n}<\phi_{k}$.

These color maps highlight the influence of convective dynamics on particle distribution in the vicinity of bluff obstacles. In addition, this comparison shows that the CDLM can be applied to model varying particle transport phenomena: ranging from purely diffusive random hopping to particulate transports including appreciable convective dynamics.

\section{Flow around an obstacle}

For the case where convective dynamics are actively considered in the CDLM, the response shown in Fig. 7(c) is representative of the hydrodynamical flow behavior observed for a disturbed fluid around a bluff obstacle in a flow channel $[25,26]$. Such flows are conventionally studied numerically with finite volume methods [27-31], cellular automata methods such as lattice gas methods (LGMs) [16,32-37] or lattice-Boltzmann methods (LBMs) [38-42], dissipativeparticle dynamics (DPD) [43-46], and multiparticle collision dynamics (MPCD) [47,48]. In the case of LGMs, LBMs, DPD, MPCD, and the CDLM presented in this study, fluid response is described on a mesoscopic length scale by coarse-graining microscopic details and retaining only those details deemed to be critical for describing fluid response.

As a case study, we now apply the CDLM to study the evolution of a particle flux beyond a bluff obstacle in a flow channel. For this configuration a wake region is expected to develop beyond the obstacle. The emergent flow patterns and their stability depends on the Reynolds number Re. When a particle flux with a maximum velocity at the center of the flow profile $U$ interacts with an obstacle with a projected width in the streamwise direction of $l$ lattice cells, Re is given by

$$
\operatorname{Re}=\frac{U l}{v} .
$$

For low Re, viscous forces dominate and no flow separation around the obstacle is observed. For higher Re fluid flows, wake formation is expected behind the obstacle develops [49-51]. The wake region is typified by the presence of a recirculation flow consisting of vortex flow patterns. Similar vortices have also been observed for particle flux around wallmounted cubic obstacles in flow channels [52-56], around geographical mountains or hills [57], around islands in shallow water [58,59], and airflows around buildings [60,61].

In this case study we examine the effect of a particle flux with varying $\mathrm{Re}$ on the velocity field in the vicinity of a square obstacle of side $l$ placed at the center of a flow channel. To simulate flows with differing values of $\mathrm{Re}$ we vary the maximum velocity in the flow $U$ by suitably adjusting the external field $A_{g, x}$. Figure 8 shows a closeup of a spatiotemporally averaged velocity field during steady state in a flow channel containing a square obstacle of side $l=300 \Delta$, particle density $\phi_{k}=0.1$, and a maximum velocity in the flow direction of $U=0.01 \Delta / \tau$. Therefore from Eq. (10) $\operatorname{Re} \approx 65$. The velocity field has been spatiotemporally averaged over an ensemble of velocity fields in steady state. The purpose of the coarse-grained velocity field is to provide a clearer representation of the macroscopic evolving flow conditions. To coarse grain the velocity field, all particles are placed in square grid blocks of side $n \Delta$, where $n$ is the number of lattice cells, according to their position vector $\mathbf{r}_{i}$. The average particle velocity $\left\langle\mathbf{v}_{j}\right\rangle$ in the $j$ th square block is then calculated using

$$
\left\langle\mathbf{v}_{j}\right\rangle=\frac{1}{N_{j}} \sum_{k=1}^{N_{j}} \mathbf{v}_{k},
$$

where $N_{j}$ is the number of particles located in the $j$ th block.

The velocity field in Fig. 8 shows the development of a recirculation zone behind the square obstacle. To highlight 


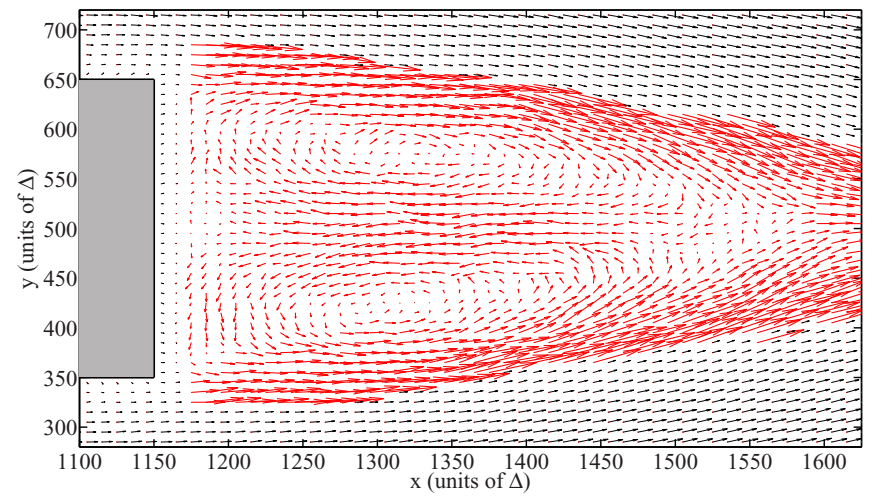

FIG. 8. Spatiotemporally averaged velocity field in a flow channel with a square obstacle of side $l=300 \Delta$ with $U=0.01 \Delta / \tau$ so that $\mathrm{Re} \approx 65$. To emphasize the recirculation zones behind the object, the arrows representing the velocity field where $\left|\left\langle\mathbf{v}_{j}\right\rangle\right| \leqslant 0.003 \Delta / \tau$ have been multiplied by a factor of two. The full dimensions of the flow channel are $L_{1}=2000 \Delta$ and $L_{2}=1000 \Delta$. This velocity field is averaged over $1.25 \times 10^{5} \tau$. For the spatial coarse graining of the lattice the cells are of size $n \Delta \times n \Delta$ where $n=10$.

the recirculation zone, velocity vectors behind the obstacle are colored in red and multiplied by a factor of two relative to the bulk velocity field if $\left|\left\langle\mathbf{v}_{j}\right\rangle\right| \leqslant 0.003 \Delta / \tau$ Within the recirculation zone we note the development of two vortices. The observation of the recirculation zones at this Reynolds number is consistent with observations from both experiments [50,51] and previous numerical simulations $[39,47,48,62]$. Figure 9 shows the corresponding color map of the average particle occupancy for the spatiotemporally averaged velocity field in Fig. 8. For the most part the particle density remains close to $\phi_{k}$, which is consistent with a smooth flux of particles through the flow channel. However, there is evidence of a slight particle build-up on the left side of the obstacle where the average local density is approximately 0.12 . It should be noted that the size of the recirculation zones in Fig. 8 are significantly larger than $n$ where $n$ is an order of magnitude larger than the maximum permitted particle move in both the convective and diffusive updates.

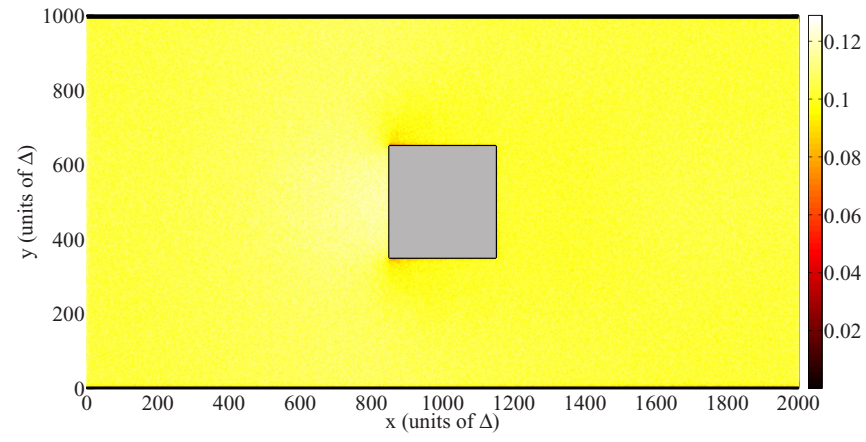

FIG. 9. Color map for average particle occupancy for a particle flow in a flow channel with a square obstacle of side $l=300 \Delta$ with $U=0.01 \Delta / \tau$ such that $\operatorname{Re} \approx 65$. The full dimensions of the flow channel are $L_{1}=2000 \Delta$ and $L_{2}=1000 \Delta$. The color map is averaged over $1.25 \times 10^{5} \tau$.

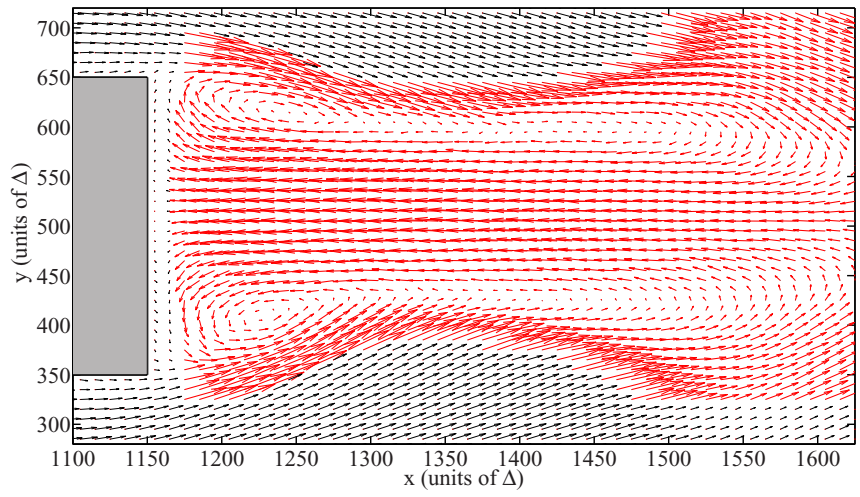

FIG. 10. Spatiotemporally averaged velocity field in a flow channel with a square obstacle of side $l=300 \Delta$ with $U=0.1 \Delta / \tau$ such that $\mathrm{Re} \approx 650$. Starting time for the velocity field is $2 \times 10^{5} \tau$, and the velocity field is averaged over a time scale of $2.5 \times 10^{4} \tau$. To emphasize the recirculation zones behind the object in each image, the arrows representing the velocity field where $\left|\left\langle\mathbf{v}_{j}\right\rangle\right| \leqslant 0.02 \Delta / \tau$ have been multiplied by a factor of two. The full dimensions of the flow channel are $L_{1}=2000 \Delta$ and $L_{2}=1000 \Delta$.

We now consider particle flows where the maximum velocity in the flow $U=0.1 \Delta / \tau$ such that the Reynolds numbers are an order of magnitude larger. Spatiotemporally averaged velocity fields around an obstacle of side $l=300 \Delta$ with $\phi_{k}=0.1$ are presented in Figs. 10 and 11. Thus from Eq. $(10), \operatorname{Re} \approx 650$. As the maximum velocity is an order of magnitude larger than for the velocity field in Fig. 8, the velocity field behind the obstacle can be resolved over a shorter averaging time scale. Figures 10 and 11 show velocity fields spatiotemporally averaged over consecutive time windows of $2.5 \times 10^{4} \tau$ where the first interval starts at $2 \times 10^{5} \tau$ (Fig. 10). Similar to the results at $\mathrm{Re} \approx 65$ presented in Fig. 8 , we observe the development of recirculation zones with vortices behind the object. Significantly, the size and position of the vortices, and the associated recirculation zone, are shown to evolve in time. For example, in Fig. 10, two distinct recirculation zones can be

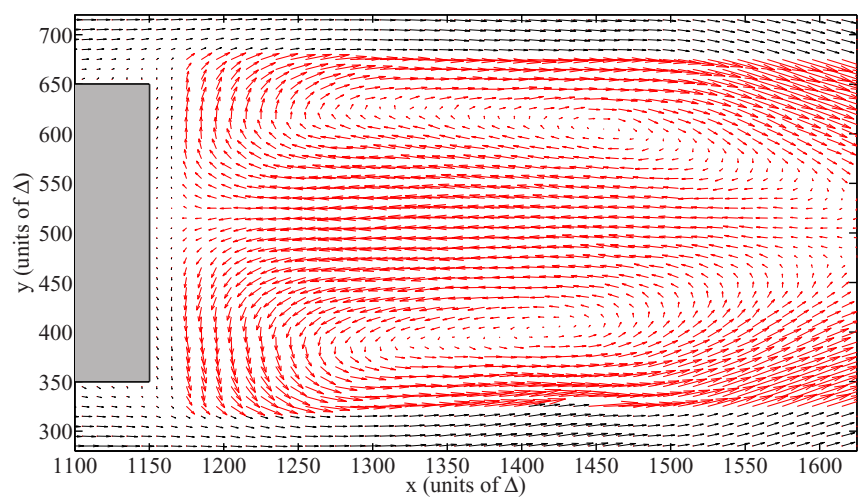

FIG. 11. Spatiotemporally averaged velocity field for the same flow channel presented in Fig. 10. Starting time for this velocity field is $2.25 \times 10^{5} \tau$, which is just after the velocity field presented in Fig. 10 . To emphasize the recirculation zones behind the object in each image, the arrows representing the velocity field where $\left|\left\langle\mathbf{v}_{j}\right\rangle\right| \leqslant 0.02 \Delta / \tau$ have been multiplied by a factor of two. The full dimensions of the flow channel are $L_{1}=2000 \Delta$ and $L_{2}=1000 \Delta$. 


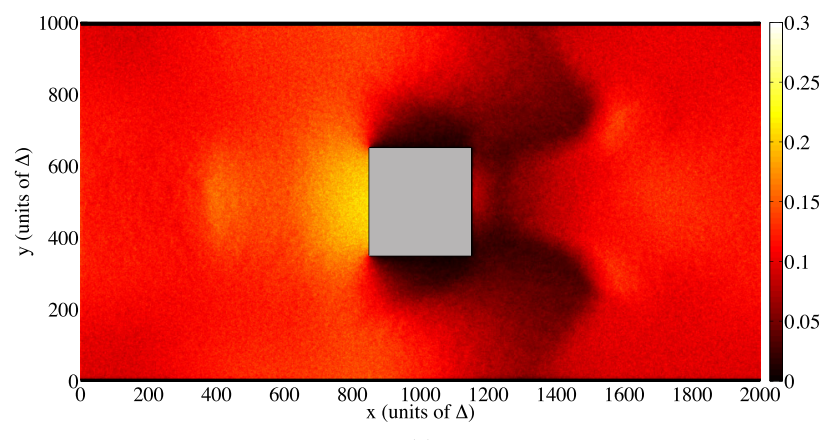

(a)

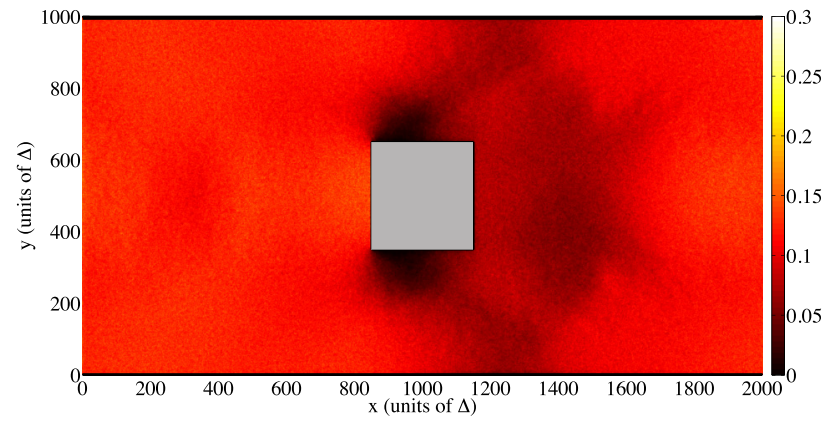

(c)

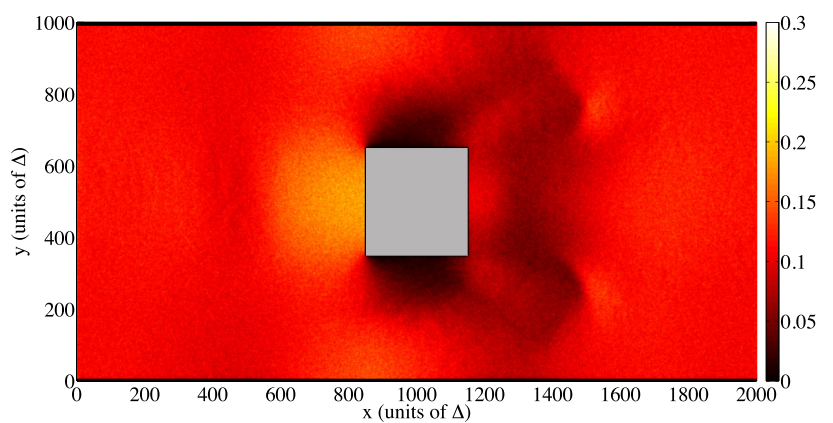

(b)

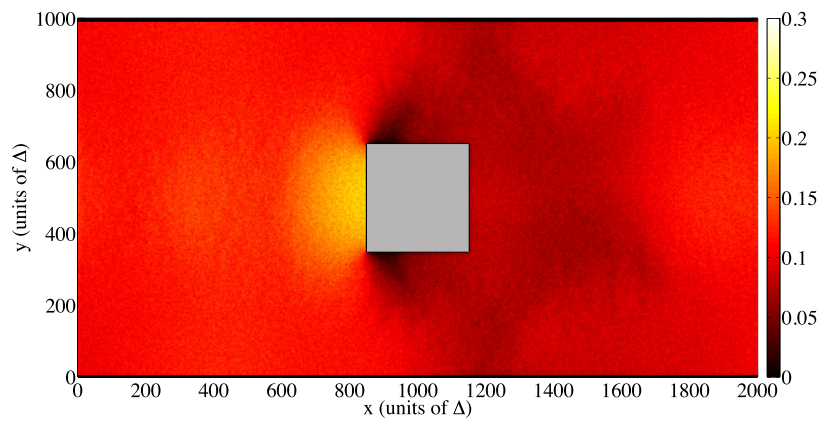

(d)

FIG. 12. Color maps for average particle occupancy in a flow channel with a square obstacle of side $l=300 \Delta$ with $U=0.1 \Delta / \tau$ such that $\operatorname{Re} \approx 650$. Each color map is averaged over a time scale of $2.5 \times 10^{4} \tau$. The starting time for each of the images is (a) $2 \times 10^{5} \tau$, (b) $2.25 \times 10^{5} \tau$, (c) $2.5 \times 10^{5} \tau$, (d) $2.75 \times 10^{5} \tau$. The full dimensions of the flow channel are $L_{1}=2000 \Delta$ and $L_{2}=1000 \Delta$.

seen adjacent to the rear of the obstacle with the centers of the recirculation zones at $x \approx 1220 \Delta$. However, for the next time window (Fig. 11), we note the absence of vortices adjacent to the obstacle and the appearance of vortices with centers at $x \approx 1450 \Delta$. This observation is indicative of the development of vortex shedding or the von Kármán vortex street $[63,64]$, where the vortices move off the back of the obstacle due to the unsteady separation of the fluid flow around the obstacle.

Figure 12 shows color maps for the average particle occupancy averaged over consecutive time windows of $2.5 \times 10^{4} \tau$. To enable direct comparison between color maps, all maps are subject to the same scaling. Each map shows the development of a wake region beyond the obstacle as well as a wake region above and below the obstacle. We also find an increase in the average particle density to the left of the obstacle in comparison to that observed for lower Re (Fig. 9). The color maps in Fig. 12 demonstrate an evolving wake region, and hence an evolving density field close to the obstacle. In Fig. 12(a) particle occupancy behind the obstacle is lowest adjacent to the rear of the obstacle. However, in Fig. 12(b) the lowest particle occupancy behind the obstacle is observed at $x \approx 1450 \Delta$. This is consistent with the approximate location of the vortices in the corresponding spatiotemporally velocity field in Fig. 11. The observation of migrating wake regions, in conjunction with an evolving velocity field, further suggests that vortex rearrangements consistent with the von Kármán vortex street have been recovered.

The spatial extent of the recirculation zone $l_{r}$ behind the obstacle is defined as the size of the closed wake or the length over which the velocities in the $x$ direction behind the obstacle are negative. Figures 13 and 14 show the axial velocity profile for flows for varying Reynolds numbers. Each axial velocity profile has been spatiotemporally averaged. For cases where $\operatorname{Re}<100$ and $U=0.01 \Delta / \tau$, the extent of the recirculation zone is clearly evident given that the local velocity field behind the object is opposite to the direction of the bulk flow field (Fig. 13). With increasing $l$, the length of the recirculation zone increases, which corresponds to trends observed in experiments [65] and previous MPCD simulations [48,66]. However, the dimensionless recirculation zone $l_{r} / l \approx 1$ at low Reynolds number (Fig. 13) which differs from previous MPCD simulations $[48,66]$. Differences in $l_{r} / l$ with previous studies may be related to the no-slip boundary conditions at

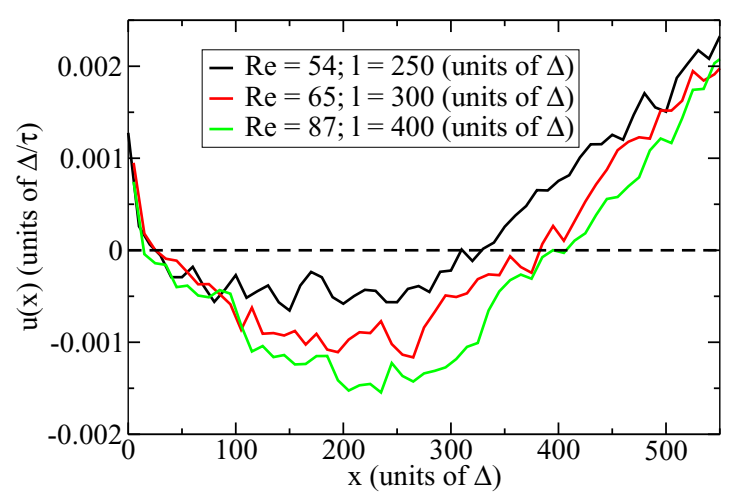

FIG. 13. Axial velocity profile $u(x)$ along the center line of the obstacle for different size obstacles and $U=0.01 \Delta / \tau$. The measure along the $x$ direction represents the distance from the right side of the obstacle. 


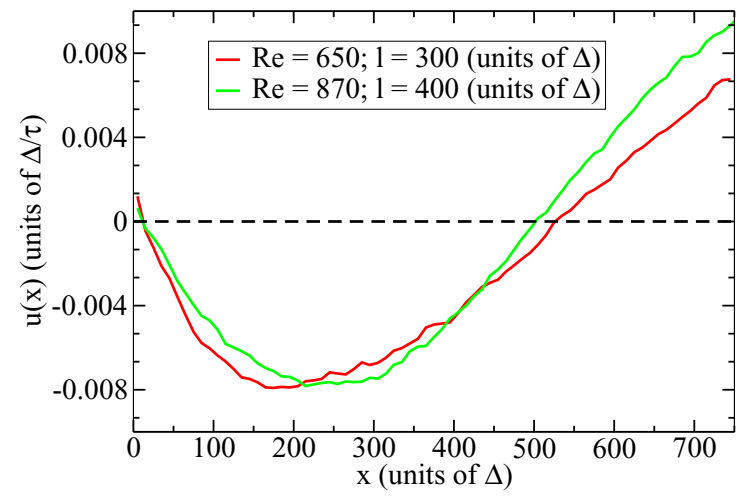

FIG. 14. Axial velocity profile $u(x)$ along the center line of the obstacle for different size obstacles and varying $U=0.1 \Delta / \tau$.

the obstacle. For all obstacle sizes, we find a positive axial velocity adjacent to the boundary. This anomaly is due to the implementation of the no-slip boundary conditions in the model where particles' velocity vectors are reversed if they attempt to penetrate the obstacle. This effect is localized to the particle layers adjacent to the obstacle as the recirculation vortices are the dominant hydrodynamic flow behavior in the wake region.

For the case where $\operatorname{Re}>100$ and $U=0.1 \Delta / \tau$ (Fig. 14), the development of a recirculation zone is dependent on the size of the square obstacle. For $l \geqslant 300 \Delta$, a recirculation zone is evident. In addition, $l_{r}$ is found to be larger with $U=0.1 \Delta / \tau$ in comparison to the same obstacle size with $U=0.01 \Delta / \tau$. For example, for $l=300 \Delta, l_{r} \approx 375 \Delta$ with $U=0.01 \Delta / \tau$ while $l_{r} \approx 535 \Delta$ with $U=0.1 \Delta / \tau$. However, with $l=250 \Delta$ we do not recover a recirculation zone when $U=0.1 \Delta / \tau$, unlike in the case where $U=0.01 \Delta / \tau$ (Fig. 13). The lack of a recirculation zone suggests that the noslip boundary conditions at the obstacle are insufficient to slow the particles enough before they enter the wake region beyond the obstacle. Recirculation zones, and thus vortices, implies the generation of angular momentum at no-slip boundaries. As the obstacle size increases, we increase the overall effect of no-slip boundary conditions on the particles and thus slow the particles adequately to motivate the development of a recirculation zone beyond the obstacle.

\section{DISCUSSION AND CONCLUSION}

In this paper, we have presented a new variant of a diffusion hopping model, the convective diffusive lattice model (CDLM). Unlike previous diffusion hopping models, it includes a description of convective particle motion which is decoupled from diffusion. Particles are restricted to discrete locations on an underlying two-dimensional square lattice. Particle dynamics are subject to an underlying velocity field which is imposed upon the same square lattice. This velocity field [Eq. (2)] is updated using an expression inspired by the compressible Navier-Stokes equation. This expression is controlled by three model parameters which account for the kinematic viscosity of the fluid, the compressibility of the fluid, and external fields such as body forces. Following the velocity field update, particle positions are updated subject to a convective update and a diffusive update which accounts for random fluctuations. During the convective update, particles are displaced from their cells subject to their velocity vector. The degree by which each particle overlaps with its neighboring cells dictates the probability of displacement to an empty neighboring lattice cell.

After calculating the diffusion coefficient for particles in the absence of convection we studied a particle flow between two no-slip boundaries leading to the development of a parabolic velocity profile consistent with laminar flow from an initial plug flow (Sec. III B). From the velocity profile we calculated the kinematic viscosity $v$ and found that $v \approx 0.046 \Delta^{2} / \tau$ (Fig. 5) with increasing system size. We then explored two specific particle flow regimes with the CDLM by comparing a flow subject to biased diffusion with a flow subject to convective dynamics and nonbiased or homogeneous diffusion. For biased diffusion a noticeable buildup of particles on the inlet side of the obstacle is observed [Fig. 7(a)] with particles predominantly traveling along two narrow channels above and below the obstacle. In addition particles rarely enter the wake region. With convective dynamics, a moderate particle buildup on the inlet side of the obstacle is observed [Fig. 7(b)]. However, particles are shown to enter the wake region, thus representing a different response in comparison to biased diffusion. Additionally the CDLM captures particle dynamics near an obstacle in a manner reminiscent of a hydrodynamic response. This study demonstrates the adaptability of the CDLM to model systems where differing particle dynamics account for specific particle transport behaviors.

As an application of the CDLM, we focused on the interaction of fluid flows with an obstacle in a flow channel for varying Reynolds numbers. To extract the macroscopic evolving flow conditions, we calculated a coarse-grained velocity field for the flow channel. For $\mathrm{Re} \approx 65$ we find a recirculation zone with two symmetric vortices (Fig. 8). However, for large Re, we find evidence of behavior consistent with the development of a von Kármán vortex street [63,64] as shown in Figs. 10 and 11. This shedding from the obstacle is due to the unsteady separation of the fluid flow around the obstacle. We also note that the size of the recirculation zone $l_{r}$ has been found to increase with Re in agreement with experiments [50,51] and previous simulations [39,47,48,62]. It is important to highlight that a recirculation zone does not develop for the case where $l=250 \Delta$ and $U=0.1 \Delta / \tau$, thus representing a flow with $\operatorname{Re} \approx 540$ (Fig. 14). This can be attributed to the importance of no-slip boundary conditions towards developing a recirculation zone. Without sufficient no-slip effects the particles do not slow down enough before entering the wake region. As a result, the particles are moving too fast to facilitate the formation of the recirculation zone. For the fluids studied here, a recirculation zone at large Re has been instigated by increasing the size of the square obstacle, and thus increasing the effect of no-slip boundary conditions. The dependency of the recirculation zone on the fluid type could also be explored in a future study with the model by varying $A_{c}$, the term associated with the compressibility of the fluid. It should be noted that in this study compressibility effects were set relatively low by choosing a sufficiently low value for $\mathbf{A}_{\mathbf{g}}$.

This initial study with the CDLM motivates further studies of flows around obstacles where diffusive and convective 
processes compete. In lattice-type models, it is relatively easy to include more complex obstacles such as circles, diamonds, or irregular geometries. In addition, the algorithm may be applied to study flow channels containing more than one obstacle. For example, the influence of one obstacle and its associated wake region on an another obstacle directly beyond the wake region could be studied as in a previous finite difference approach [67]. It is also conceivable to place a regular array of obstacles in the flow channel to study the effect of multiple obstacles on flow and diffusion characteristics as in simulations on plume dispersion through objects [68], heat transfer in flows around a matrix of all mounted objects [69], or to size separate biological particles and DNA molecules [70].

The model could also be applied to study flows in simple porous media represented as a series of obstacles of varying geometries within a flow channel. The FHP lattice gas model [16] has already been applied to study flow throw a simplistic two-dimensional porous medium [37] and subsequently expanded to three-dimensional systems [36,41]. Recently, LBMs have been applied to study high Reynolds number flows around single circular obstacles and a porous medium represented as a collection of these obstacles [39] and coupled with a discrete-element method to study the effect of porosity on soil permeability [42]. The CDLM presented here is an advancement on diffusion hopping models and lattice-type models in that it incorporates both diffusive and convective particle dynamics. Therefore, the model is well placed to be used in the study of flows in simple porous media in addition to other flows where a description of hydrodynamic flow behaviors is a necessity. We also envisage applications in active matter systems where collective dynamics greatly influences system behavior.

\section{ACKNOWLEDGMENTS}

B.W.F. acknowledges financial support from the "Over Grenzen" programme of KNAW (Royal Dutch Academy of Arts and Sciences). J.T.P. acknowledges financial support from the European Research Council (ERC), under its Consolidator Grant scheme, contract no. 615906 (NonSphereFlow). R.v.S. gratefully acknowledges support from the Netherlands Gravitation program Center "Multiscale Catalytic Energy Conversion (MCEC)."
[1] J.-P. Bouchaud and A. Georges, Phys. Rep. 195, 127 (1990).

[2] J. P. Gonzalez-Vazquez, J. A. Anta, and J. Bisquert, Phys. Chem. Chem. Phys. 11, 10359 (2009).

[3] V. M. Burlakov, K. Kawata, H. E. Assender, G. A. D. Briggs, A. Ruseckas, and I. D. W. Samuel, Phys. Rev. B 72, 075206 (2005).

[4] I. Nachev, J. Deppe, R. Wallis, and M. Balanski, Mater. Sci. Eng. B 15, 260 (1992).

[5] J. Deppe, R. Wallis, I. Nachev, and M. Balkanski, J. Phys. Chem. Solids 55, 759 (1994).

[6] L.-H. Cai, S. Panyukov, and M. Rubinstein, Macromolecules 48, 847 (2015).

[7] P.-C. Jiang, T.-Y. Yu, W.-C. Perng, and L.-P. Hwang, Biophys. J. 80, 2493 (2001).

[8] K. Nagel, Phys. Rev. E 53, 4655 (1996).

[9] D. Chowdhury, D. E. Wolf, and M. Schreckenberg, Physica A 235, 417 (1997).

[10] K. Nagel, Transp. Res. Record 1644, 1 (1998).

[11] D. Andreucci, D. Bellaveglia, and E. N. M. Cirillo, Math. Biosci. 257, 42 (2014).

[12] D. Andreucci, D. Bellaveglia, E. N. M. Cirillo, and S. Marconi, Discret. Contin. Dyn. Syst. Ser. B 19, 1837 (2014).

[13] E. A. Codling, M. J. Plank, and S. Benhamou, J. R. Soc. Interface 5, 813 (2008).

[14] E. N. Cirillo, O. Krehel, A. Muntean, R. van Santen, and A. Sengar, Physica A 442, 436 (2016).

[15] J. Hardy, O. de Pazzis, and Y. Pomeau, Phys. Rev. A 13, 1949 (1976).

[16] U. Frisch, B. Hasslacher, and Y. Pomeau, Phys. Rev. Lett. 56, 1505 (1986).

[17] J. J. Monaghan, Ann. Rev. Astron. Astrophys. 30, 543 (1992).

[18] J. J. Monaghan, Rep. Prog. Phys. 68, 1703 (2005).

[19] M. Ellero, M. Serrano, and P. Español, J. Comput. Phys. 226, 1731 (2007).
[20] L. D. G. Sigalotti, J. Klapp, E. Sira, Y. Meleán, and A. Hasmy, J. Comput. Phys. 191, 622 (2003).

[21] J. P. Morris, P. J. Fox, and Y. Zhu, J. Comput. Phys. 136, 214 (1997).

[22] M. Mourao, D. Kreitman, and S. Schnell, Phys. Chem. Chem. Phys. 16, 4492 (2014).

[23] D. Ben-Avraham and S. Havlin, Diffusion and Reactions in Fractals and Disordered Systems (Cambridge University Press, Cambridge, 2000).

[24] J. Krug, Phys. Rev. Lett. 67, 1882 (1991).

[25] M. M. Zdravkovich, Flow Around Circular Cylinders, Vol. 1: Fundamentals (Oxford University Press, Oxford, 1997).

[26] M. M. Zdravkovich, Flow Around Circular Cylinders, Vol. 2: Applications (Oxford University Press, Oxford, 2003).

[27] J. Bernsdorf, F. Durst, and M. Schäfer, Int. J. Numer. Met. 29, 251 (1999).

[28] D. R. Noble, J. G. Georgiadis, and R. O. Buckius, Int. J. Numer. Met. 23, 1 (1996).

[29] F. Nicoud, J. Comput. Phys. 158, 71 (2000).

[30] J. Kim, D. Kim, and H. Choi, J. Comput. Phys. 171, 132 (2001).

[31] B. Xie, S. Ii, A. Ikebata, and F. Xiao, J. Comput. Phys. 277, 138 (2014).

[32] D. H. Rothman and S. Zaleski, Rev. Mod. Phys. 66, 1417 (1994).

[33] B. W. Fitzgerald, I. Clancy, and D. Corcoran, Physica A 410, 582 (2014).

[34] A. Károlyi and J. Kertész, Comp. Phys. Comm. 121-122, 290 (1999).

[35] G. Peng and H. J. Herrmann, Phys. Rev. E 49, R1796 (1994).

[36] P. Papatzacos, Complex Syst. 3, 383 (1989).

[37] D. H. Rothman, Geophysics 53, 509 (1988).

[38] S. Chen and G. D. Doolen, Annu. Rev. Fluid Mech. 30, 329 (1998).

[39] A. Grucelski and J. Pozorski, Comp. Fluids 71, 406 (2013).

[40] Z. Guo and T. S. Zhao, Phys. Rev. E 66, 036304 (2002). 
[41] S. Succi, E. Foti, and F. Higuera, Europhys. Lett. 10, 433 (1989)

[42] B. Sheikh and A. Pak, Phys. Rev. E 91, 053301 (2015).

[43] P. J. Hoogerbrugge and J. M. V. A. Koelman, Europhys. Lett. 19, 155 (1992).

[44] J. M. V. A. Koelman and P. J. Hoogerbrugge, Europhys. Lett. 21, 363 (1993).

[45] E. Moeendarbary, T. Y. Ng, and M. Zangeneh, Int. J. Appl. Mech. 01, 737 (2009)

[46] P. Español and P. Warren, Europhys. Lett. 30, 191 (1995).

[47] A. Lamura, G. Gompper, T. Ihle, and D. M. Kroll, Europhys. Lett. 56, 319 (2001).

[48] A. Lamura and G. Gompper, Eur. Phys. J. E 9, 477 (2002).

[49] G. Birkhoff, J. Appl. Phys. 24, 98 (1953).

[50] D. J. Tritton, J. Fluid Mech. 6, 547 (1959).

[51] M. Contanceau and R. Bouard, J. Fluid Mech. 79, 231 (1977).

[52] J.-Y. Hwang and K.-S. Yang, Phys. Fluids 16, 2382 (2004).

[53] A. Liakos and N. A. Malamataris, Phys. Fluids 26, 053603 (2014).

[54] A. van Dijk and H. C. de Lange, Comput. Fluids 36, 949 (2007).

[55] A. Sau, R. R. Hwang, T. W. H. Sheu, and W. C. Yang, Phys Rev. E 68, 056303 (2003).

[56] A. Yakhot, T. Anor, H. Liu, and N. Nikitin, J. Fluid Mech. 566, 1 (1996).
[57] G. P. Almeida, D. F. G. Durão, and M. V. Heitor, Exp. Therm. Fluid. Sci. 7, 87 (1993).

[58] P. K. Stansby and P. M. Lloyd, J. Fluid Mech. 429, 239 (2001).

[59] P. M. Lloyd, P. K. Stansby, and D. Chen, J. Fluid Mech. 429, 217 (2001).

[60] Y.-H. Tseng, C. Meneveau, and M. B. Parlange, Environ. Sci. Technol. 40, 2653 (2006).

[61] X. Cheng and F. Hu, Adv. Atmos. Sci. 22, 290 (2005).

[62] M. Cheng, D. Whyte, and J. Lou, J. Fluids Structures 23, 207 (2007).

[63] T. von Kármán, Nachf. Ges. Wissenschaft. Göttingen, 507 (1911).

[64] T. von Kármán, Nachf. Ges. Wissenschaft. Göttingen, 547 (1912).

[65] M. Breuer, J. Bernsdorf, T. Zeiser, and F. Durst, Int. J. Heat Fluid Flow 21, 186 (2000).

[66] E. Allahyarov and G. Gompper, Phys. Rev. E 66, 036702 (2002).

[67] P. P. Patil and S. Tiwari, Eng. Appl. Comp. Fluid 3, 369 (2009).

[68] M. J. Davidson, W. H. Snyder, R. E. Lawson Jr., and J. C. R. Hunt, Atmos. Environ. 30, 3715 (1996).

[69] E. Meinders and K. Hanjalić, Int. J. Heat Fluid Flow 20, 255 (1999).

[70] N. Pamme, Lab Chip 7, 1644 (2007). 